\title{
Early Trauma
}

\section{Viorica Lazin*}

Counseling and Advanced Human Studies, USA

*Corresponding Author: Viorica Lazin, Counseling and Advanced Human Studies, USA.

Received: August 26, 2019; Published: September 13, 2019

DOI: 10.31080/ASNE.2019.02.0106

Early trauma or Post Traumatic Disorder (PSTD), Emotional Deprivation (ED), Attachment Disorders (AD), Reactive Attachment Disorders (RAD), Emotional Trauma (ET), Somatoform Emotional Disorders (SED), Adjustment Disorder Symptoms (ADS) are all these clinical entities, lacking clear delineating parameters, used indiscriminately confusing mental health and medical professionals, and parents [1]. Granted these cognitive-emotional-affective and behavioral complexities often are overlapping, puzzling at time clinical diagnosticians, fact evident that even the newest Diagnostic Statistical Manual-V (DSM-V) had been elucidating the matters [1]. Although, there were sporadic attempts to address such confusion, although universal acceptance is needed, as one cannot proper treat if one does not correctly understand [2]. Early emotional trauma was the topic of the presentation. Mental health professionals recognize that early emotional deprivation scars, and the consequences are an emotional-affective disorder, attachment disorder, impulsive obsessive disorder, attention deficit and hyperactivity disorder, posttraumatic disorder, low self-esteem, depression, schizophrenia, and various addictions and other mental health challenges. Understanding the early trauma and its echoes on the brain and nervous system are coping mechanisms, where the body is attempting to adapt to such traumatic events, trying and/or making sense, adapting in attempt to survive. The learning objective of this papers is to facilitate an understanding what trauma is, what do we know about early trauma, how many traumas are, impact of trauma on the development, neurobiology of the trauma and its translation on the brain, body, senses, and feelings and lastly promoting resilience and healing.

An effective teacher knows that learning must be a creative and dynamic process that is also empowering; it is in fact an a new information that needs to be tailored to be a compatible interchangement of a new information that needs to be built on students' past experience- only in that way the leaning occurs, when the learning becomes a process of integrating the new learned concepts that resonate well with the past leanings, learning acquired by students though the past experiences $[3,4]$. Nelson and Neufeld [4] pointed that at the heart of imparting knowledge is a relationship between teacher and student wherein the teacher can effectively communicate knowledge and the student able to internalize and activate the teachings. Hence, my learner-oriented approach of presenting information, engaging audience, to take part in the learning process, to experiment the learning [5].

\section{Experiential learning}

Experiential learning is imparting a new knowledge connected with the existing knowledge, facilitating this process by appealing to various senses such as sight, hearing and feeling [6]. A process of learning that is appeals to more than one senses, such as visual and auditory, becomes more palatable suggestive. A pertinent pictorial representations coupled with two other professional multimedia presentations becomes more palatable to the audience to be able to understand how highly subjective the trauma is, a professor can invite students to an everyday experience, by inviting the audience to be relax, inducing such relaxed atmosphere by means of a soft music, that is abruptly interrupted by a harsh strongly annoying sound. Inviting the students to engage in discussions so as to process that experience, guiding the discussion to highlight how subjectively unique is the sensorial quality of trauma.

\section{Fostering knowledge}

Understanding and recognizing various early childhood traumas from infancy to preschool had not been facilitated by the above authors. It is a well-recognized fact that trauma deeply impacts the neurobiological development heavily taxing emotional- affective moods, the brain and the body, along with perceptions of five senses and sensorimotor integration [7-9]. Same authors agreed that infants' effects of such traumatic effects are brain and body underdevelopment, emotional-affective labile and/or frozen affect, incapacity to communicate and or feel, while adults effects are chronic major depressive disorder and anxieties, various addictions, obsessive compulsive disorders, bipolar, schizophrenia and psychotic disorders, fatigue, poor immune functions, eating disorders and obesity, hypertension, interpersonal difficulties, aggressive beha- 
vior, low self-esteem, depression, anxiety. Looking at the trauma from various lenses, such as cognitive behavioral, neurodevelopmental and how understanding of lens of trauma would help identify the elements of effective intervention and why they work and apply a range of play based sensory processing interventions to a comprehensive treatment and recovery program. The relationship between an infant and his or her primary caregivers literally builds and shapes the brain, which in turn organizes the growth of the physical, cognitive, and emotional systems [7]. Healing from the early trauma can be attained by building healthy attachments. Healthy attachments are relationships that develop from interactions between the caregiver and infant and understanding a few basics about how the brain works one is better equipped to understand a child, to respond more effectively to difficult situations, build a foundation for social and emotional growth. However, the baby who is roughly or inconsistently handled, without having appropriate boundaries, develops a more reactive stress response with different biochemical pathways and signals that have life-long implications for health and learning [7-9]. Using sequenctial multisensorial therapies and intervetnions can faciliate healing from trauma.

\section{Skill building}

Early trauma leads to fear and hyperarousal as unconscious negative coping reactions, which in return trigger continuous chronic neurohormonal brain activation of hypothalamic-pituitary-adrenal (HPA) axis in which cortisol stress hormone reaches such high stimulation in brain's emotional centers such as hypothalamic hippocampus until it becomes exhausted [10]. This translates in permanent fear acclimatization and inattention, impulse control, sleep and fine motor problems, even when conditions change in a more positive [10]. The heightened arousal state leads to a highly sensitization to nonverbal clues such as eye contact, touch of arm, which instantaneously are translated as threats, due to the intense disruption or arresting of any positive emotional attachment [10]. Disruptions and arresting of positive emotional attachments resulted in increased susceptibility to stress, inability to regulate and properly read and understand emotions and excessive help seeking and dependency or excessive social isolation- such changes arrest one's resilient capacities, paving the road to various addictions, addictions that are not but attempts of coping with the stress and emotional deprivation traumas [10]. Understanding various early traumas and their effects on neuron-hormonal and sensorial development, and how these translate on one's cognitive, emotional and behavioral makeup were some important learning objectives. Understanding the need of therapy and parenting quality, evaluating the efficacy of the positive therapeutic and parenting interventions, based on stimulation of what TLC advocates for the Structured Sensory Intervention for Traumatized Children,
Adolescents and Parents (SITCAP), which is based on structured sensory therapy, integrating sensory-based activities and cognitive-reframing strategies [11-13].

\section{Conclusion}

In conclusion, trauma is a multidimensional complex topic; a topic that needs much more understanding. Hence the need to educate, to raise awareness, hence it needs to be more often presented to the public, to facilitate not only an understanding, but also to acknowledge the pervasiveness of the trauma, that crosses political, geographical, cultural boundaries, being universal to all, and therefore there is an imperious need to focusing on trauma's healing methods, teaching communities to foster positive resilience and healing from trauma, so as to avoid resorting to addictions as means of coping with traumas.

\section{Bibliography}

1. Grohol J. "Adjustment Disorder Symptoms". Psychcentral (2013).

2. Egger HL and Emde RN. "Developmentally-Sensitive Diagnostic Criteria for Mental Health Disorders in Early Childhood: the Diagnostic and Statistical Manual of Mental Disorders-IV, the Research Diagnostic Criteria-Preschool Age, and the Diagnostic Classification of Mental Health and Developmental Disorders of Infancy and Early Childhood-revised". American Psychology 66.2 (2011): 95-106.

3. Harris A. "Effective teaching: A review of the literature". School Leadership and Management 18.2 (1998): 169-183.

4. Nelson ML and Neufeldt SA. "The pedagogy of counseling: A critical examination". Counselor Education and Supervision 38.2 (1998): 70-89.

5. Brookfield S D. "Understanding and facilitating adult learning”. San Francisco: Jossey-Bass. (1986).

6. Grant J. "Training counselors to work with complex clients: Enhancing emotional responsiveness through experiential methods". Counselor Education and Supervision, 45.3 (2006): 218-230.

7. American Psychiatric Publishing. "Highlights of Changes from DSM-IVTR to DSM-5". American Psychiatric Association (2013).

8. National Scientific Council on the Developing Child [NSCDC] (2004).

9. The Michigan Child Welfare Law [MCWL]. "State Bar of Michigan Children Law Section 13.2 (2010). 
10. Child Welfare Information Gateway. "Understanding the Effects of Maltreatment on Brain Development". Issues Briefs, U.S. Department of Health and Human Services (2009).

11. Anderson R D and Price G E. "Experiential groups in counselor education: Student attitudes and instructor participation". Counselor Education and Supervision 41.2 (2001): 111-119.

12. Hubbs DL and Brand CF. "The paper mirror: Understanding reflective journaling". Journal of Experiential Education 28.1 (2005): 60-71.

13. Childhood Trauma.

Volume 2 Issue 10 October 2019

(C) All rights are reserved by Viorica Lazin. 